Beim Sozialgericht setzte man sich mit der Frage auseinander, ob der Kalligraf bei seinem Schaffen über ein "Mindestmaß an freier schöpferischer Gestaltung" verfügt. Nur wer in einem solchen Mindestmaß schöpferisch gestalten kann, so das Bundessozialgericht in seiner langjährigen Rechtsprechung, könne Künstler sein im Sinne des § 2 Satz 1 KSVG. Dies hatte die KSK verneint mit dem Argument, dass der Kalligraf ja nur Buchstaben ausschmücke, aber an die Grundform gebunden sei. Die Gegenfrage, warum dann deutsche Typografen als Künstler anerkannt werden, blieb unbeantwortet - obwohl oder weil der Gestaltungsspielraum eines Kalligrafen weit größer ist als der eines Typografen. In der mündlichen Verhandlung ließen sich die Richter Beispiele zeigen und kamen zu dem Schluss, dass der Kläger durchaus über einen eigenschöpferischen Gestaltungsspielraum verfüge und die Buchstaben ohne bindende Vorgaben ausschmücke. Außerdem sei er auch in Fachkreisen anerkannt (was nur relevant ist für den Fall, dass man inn nicht als Künstler, sondern als Kunsthandwerker einstufen wollte).

Die KSK hingegen überzeugte dies nicht, sie blieb weiter bei ihrer Beobachtung, dass nach allgemeiner Verkehrsauffassung in Deutschland arabische Kalligrafie als Nichtkunst eingestuft werde. Auch die Frage, woher diese Kenntnis stamme, wurde nicht beantwortet, und so legte die KSK Sprungrevision zum Bundessozialgericht ein. Ihr wesentliches Argument: der Kläger sei kein Künstler, sondern Kunsthandwerker und habe die nötige Anerkennung in künstlerischen Fachkreisen nicht nachgewiesen. Der Kläger sei auch nicht einem Illustrator oder Grafiker vergleichbar. Mithin sei die Versicherungspflicht zu verneinen.

Das Bundessozialgericht griff auf seine jahrelange Rechtsprechung zur Definition der "Kunst“ im Sinne des § 2 Satz 1 KSVG zurück. Der Begriff sei aus dem Regelungszweck des KSVG und unter Berücksichtigung der allgemeinen Verkehrsauffassung und der historischen Entwicklung zu bestimmen. Ob die Ursprünge der zu beurteilenden Kunstform dabei im Ausland liegen, ist nach der ausdrücklichen Feststellung der Richter unerheblich.
Die Argumentation des Klägers war für sich genommen sehr schlicht: Kalligrafie - ob europäische, japanische oder arabische - gehört zur grafischen Kunst und damit zur bildenden Kunst iSd § 2 Satz 1 KSVG. Wenn schon der Typograf - zu Recht - als Künstler im Sinne der KSK anerkannt wird, dann muss erst recht ein Kalligraf künstlerisch tätig sein, der die Buchstaben individuell zu einer Grafik ausschmückt. Der Kläger hatte beispielsweise den Schriftzug des arabischen Grußes „salam aleikum“ dreifach dargestellt: zu einem Kreis geformt in der Mitte der Grafik und in zwei Ringen um den Kreis herum - ein Symbol für die Ebenen des Handelns, des Herzens und des Gottes.

Die KSK hatte sich auch in der mündlichen Verhandlung allein auf die Frage gestützt, ob der Kläger als Kunsthandwerker in künstlerischen Fachkreisen anerkannt ist. Auf die Frage des vorsitzenden Richters, welche Argumente denn gegen die Einordnung der Kalligrafie als originär grafische Kunst angeführt werden können, konnte der KSK-Vertreter keine Antwort geben.

Anders das Gericht: es hat differenziert und festgestellt, dass Kalligrafie sich auf ein reines Kunsthandwerk beschränken kann, wenn nämlich die manuell-technischen Fertigkeiten zur Schreibung nach vorgegebenen Schriftzeichen im Vordergrund stehen. Kalligrafie sei jedoch dann Kunst im Sinne des KSVG, wenn "den hergestellten Kalligrafien überwiegend ein hohes Maß an eigenschöpferischen Inhalten zukommt, so dass sie als ein von dem zugrunde liegenden Schriftzeichen losgelöstes eigenes künstlerisches Werk (...) zu bewerten ist."

Nach den für das BSG bindenden Tatsachenfeststellungen und der eigenen Anschauung anhand der vorgelegten Beispiele - verfügt der Kläger über einen solchen eigenschöpferischen Gestaltungsspielraum. Die Versicherungspflicht des Kalligrafen war damit anzuerkennen. Und es bleibt die Frage, warum dieser Prozess überhaupt notwendig war.

\title{
Rechtsprechung
}

\section{Zur Künstlereigenschaft des Kalligrafen}

Bundessozialgericht, Urteil vom 15. November 2007 - B 3 KS 3/07 R

\section{Ein Kalligraf, der von einem zugrunde liegenden Schriftzeichen losgelöste Bilder und Grafiken mit eigenständiger Identität und Aussagekraft schafft, ist Künstler im Sinne des Künstlersozialversicherungsgesetzes (KSVG).}

\section{Tatbestand}

- Streitig ist dieVersicherungspflicht des Klägers nach dem Künstlersozialversicherungsgesetz (KSVG) für seine Tätigkeit als Kalligraf.

Der 1957 in Kairo geborene Kläger hat von 1980 bis 1986 als Dekorateur für Metall- und Holzornamente in verschiedenen arabischen Staaten gearbeitet und war im Anschluss daran in
Deutschland zunächst als Koch beschäftigt. Seit 1999 übt er eine selbstständige Tätigkeit als Kalligraf aus und verdient hierdurch eigenen Angaben zufolge rund 4.000 Euro pro Jahr. Zunächst betrieb der Kläger die Kalligrafie überwiegend als Raumgestaltung, später hat er sich dann stärker grafisch ausgerichtet. Auf Märkten bietet er an, Namen in arabischer Kunstschrift zu schreiben und Glückwünsche, Segenssprüche oder Logos zu gestalten. Zudem hat er seine Werke in einer Galerie ausgestellt und 
Originale, Drucke sowie Postkarten in einer Fachbuchhandlung für Islam und Orient präsentiert und verkauft.

Den Antrag des Klägers vom 3. April 2003 auf Feststellung der Versicherungspflicht nach dem KSVG lehnte die Beklagte mit der Begründung ab, als Kalligraf übe er keinen künstlerischen Beruf aus; es handele sich vielmehr um eine Gestaltung von Texten, denn auch in der arabischen Schrift dürfe der einzelne Buchstabe nur unwesentlich gestalterisch verändert werden (Bescheid vom 1. August 2003 in Gestalt des Widerspruchsbescheids vom 20. Oktober 2003).

Das Sozialgericht (SG) hat der hiergegen gerichteten Klage stattgegeben und unter Aufhebung der angefochtenen Bescheide festgestellt, dass der Kläger als Kalligraf ab 3. April 2003 der Versicherungspflicht nach dem KSVG unterliegt (Urteil vom 14. November 2006). Der Kläger sei Künstler, weil sich in seinen Werken eine eigenschöpferische Leistung zeige. Zwar verwende er jeweils einen arabischen Schriftzug als Grundlage seiner Bilder, wandele diesen jedoch individuell so ab, dass ein eigenständiges und über das reine Schönschreiben des Schriftzeichens hinausgehendes Kunstwerk entstehe. Zudem habe der Kläger eine Ausstellung in einer Galerie sowie die wiederholte Teilnahme an einem Handwerker- und Künstlermarkt nachgewiesen; seine Tätigkeit sei der eines Malers oder Bildhauers vergleichbar.

Hiergegen richtet sich die Sprungrevision der beklagten Künstlersozialkasse. Die Tätigkeit des Kalligrafen sei nicht im Künstlerbericht der Bundesregierung von 1975 verzeichnet, obwohl es sich um eine damals schon bekannte Betätigung gehandelt habe; bereits das spreche gegen ihre Einordnung als künstlerische Tätigkeit. Der Kläger sei auch weder einem Maler noch einem Zeichner oder künstlerischen Grafiker vergleichbar. Deshalb sei darauf abzustellen, ob der Betreffende in einschlägigen fachkundigen Kreisen als gleichwertiger Künstler angesehen werde; daran mangele es vorliegend. Im Übrigen sei grundsätzlich nur die inländische Verkehrsauffassung maßgeblich; die aus der islamischen Welt stammende Tätigkeit als Kalligraf falle deshalb nicht in den Schutzbereich des KSVG. [...]

\section{Entscheidungsgründe}

Die Revision der Beklagten ist unbegründet. Der Kläger ist zumindest seit Antragstellung am 3. April 2003 als Künstler nach dem KSVG versicherungspflichtig tätig. Das SG hat deshalb im Ergebnis zu Recht den Bescheid der Beklagten vom 1. August 2003 in Gestalt des Widerspruchsbescheids vom 20. Oktober 2003 aufgehoben und festgestellt, dass der Kläger der Versicherungspflicht nach dem KSVG unterliegt.

1. Rechtsgrundlage des Begehrens auf Feststellung der Versicherungspflicht in der Künstlersozialversicherung ist $§ 1 \mathrm{Nr}$. 1 KSVG. Danach werden selbstständige Künstler und Publizisten in der Rentenversicherung der Angestellten, in der gesetzlichen Kranken- und in der sozialen Pflegeversicherung versichert, wenn sie eine künstlerische oder publizistische Tätigkeit erwerbsmäßig und nicht nur vorübergehend ausüben. Nach den für den Senat bindenden ( $\S \S$ 161 Abs. 4, 163 SGG) Tatsachenfeststellungen des SG liegen diese
Voraussetzungen hier vor; als arabischer Kalligraf übt der Kläger eine künstlerische Tätigkeit iSd KSVG aus.

a) Als künstlerische Tätigkeit werden in § 2 Satz 1 KSVG drei Bereiche jeweils in den Spielarten des Schaffens, Ausübens und Lehrens umschrieben, nämlich die Musik, die Bildende Kunst und die Darstellende Kunst. Eine weitergehende Festlegung, was darunter im Einzelnen zu verstehen ist, ist im Hinblick auf die Vielfalt, Komplexität und Dynamik der Erscheinungsformen künstlerischer Betätigungsfelder nicht erfolgt. Der Gesetzgeber spricht im KSVG nur allgemein von „Künstlern“ und „künstlerischen Tätigkeiten“, auf eine Definition des Kunstbegriffs hat er hingegen bewusst verzichtet (BT-Drucks 8/3172, S 21). Dieser Begriff ist deshalb aus dem Regelungszweck des KSVG unter Berücksichtigung der allgemeinen Verkehrsauffassung und der historischen Entwicklung zu erschließen (vgl. BSG SozR 4-5425 § 24 Nr. 6 Rn. 13 und BSGE 83, 160, $161=$ SozR 3-5425 § $2 \mathrm{Nr} .9 \mathrm{~S} 33$ - jeweils mwN; zum Kunstbegriff des Art. 5 GG vgl. BVerfGE 30, 173, 188 ff. und 81, 108, 116; zur Zielrichtung des KSVG vgl. BT-Drucks. 9/26, S 18 und BT-Drucks. 8/3172, S 19 ff.). Aus den Materialien zum KSVG ergibt sich, dass der Begriff der Kunst trotz seiner Unschärfe auf jeden Fall solche künstlerischen Tätigkeiten umfasst, mit denen sich der „Bericht der Bundesregierung über die wirtschaftliche und soziale Lage der künstlerischen Berufe (Künstlerbericht)" aus dem Jahre 1975 (BT-Drucks. 7/3071) beschäftigt (BSGE 83, 160, 165 f. = SozR 3-5425 § 2 Nr. 9 S. 37 f; BSGE 83, 246, 250 = SozR 3-5425 § 1 Nr. 5 S. 23; vgl. auch Finke/Brachmann/Nordhausen KSVG, 3. Aufl. 2004, § 2 Rn. 3 und 9; Schriever Der Begriff der Kunst im Künstlersozialversicherungsrecht, in: von Wulffen/Krasney (Hg.), FS 50 Jahre Bundessozialgericht, 2004, S. 709, 714 f. ). Der Gesetzgeber hat damit einen an der Typologie von Ausübungsformen orientierten Kunstbegriff vorgegeben, der in aller Regel dann erfüllt ist, wenn das zu beurteilende Werk den Gattungsanforderungen eines bestimmten Kunsttyps entspricht. Bei diesen Berufsfeldern ist das soziale Schutzbedürfnis zu unterstellen, ohne dass es auf die Qualität der künstlerischen Tätigkeit ankommt oder eine bestimmte Werk- und Gestaltungshöhe vorausgesetzt wird (BSG aaO).

b) Der Qualifizierung der Kalligrafie als künstlerische Tätigkeit iSd Vorschriften steht nicht entgegen, dass sie im oben angeführten Künstlerbericht der Bundesregierung als Kunsttyp nicht erwähnt wird. Die Nichterwähnung einer Tätigkeit im Künstlerbericht spricht zwar dafür, dass es jedenfalls zur Zeit seiner Erstellung keine allgemeine Verkehrsauffassung in Deutschland gab, diese Tätigkeit als künstlerisch einzuordnen (vgl. Urteil des Senats vom 28. Februar 2007 - B 3 KS 2/07 R-,zurVeröffentlichung vorgesehen-Tätowierer). Jedoch hat der Senat bereits entschieden, dass der Künstlerbericht einer solchen Einordnung dann nicht entgegensteht, wenn es die Tätigkeit zur Zeit seiner Erstellung noch gar nicht gegeben hat (vgl. BSG SozR 4-5425 § 2 Nr. 5 zur Künstlereigenschaft von Webdesignern). Entsprechendes gilt, wenn sich nach der Erstellung des Künstlerberichts aus dem Jahre 1975 die Verkehrsauffassung hinsichtlich einer früher bereits bekannten Tätigkeit grundlegend gewandelt hat oder wenn der betreffende Kunsttyp von einer so kleinen Gruppe von Kunstschaffenden ausgeübt wird, dass er bei der Einordnung in die Kunstgattungen des Künstlerberichts außer Betracht bleiben konnte. Würde der Künstlerbericht hingegen auch solche, nur in einem marginalen Umfang aus- 
geübte Gattungen ausschließen, so würde dies dem angesichts der Vielfalt und Dynamik in der Entwicklung künstlerischer Betätigungen bewusst offen gehaltenen Kunstbegriff des $\S 2$ KSVG widersprechen (vgl. die Gesetzesmaterialien zum KSVG, BT-Drucks. 8/3172, S. 21, und 9/26, S. 18). In solchen Fällen ist ausgehend vom Künstlerbericht mit seinen Katalogberufen als Einordnungshilfe vielmehr selbstständig nachzuvollziehen, ob die zu beurteilende Tätigkeit nach den für die Aufstellung des Künstlerberichts maßgebenden Kriterien einem der drei Bereiche künstlerischer Tätigkeit zuzuordnen ist und ob sie weder als Traditions- und Brauchtumspflege (BSG SozR 4-5425 § 2 Nr. 3 - japanische Teezeremonie) noch als (kunst)handwerkliche Tätigkeit (vgl. Urteil des Senats vom 28. Februar 2007 - B 3 KS 2/07 R - Tätowierer, zur Veröffentlichung in BSGE und SozR vorgesehen; BSGE 80, $136=$ SozR 3-5425 § 2 Nr. 5 - Musikinstrumentenbauer; BSGE 82, 164 = SozR 3-5425 § 2 Nr. 8 - Feintäschner) aus dem Schutzbereich des KSVG auszugrenzen ist.

c) Unbeachtlich für die auf dieser Grundlage vorzunehmende Prüfung ist, ob die zu beurteilende Kunstgattung Ursprünge im Ausland oder fremdländischeWurzeln besitzt. Der Inlandsbezug des KSVG schließt nicht aus, dass auch derjenige selbstständige Künstler Versicherungsschutz nach dem KSVG genießt, der eine Kunstform iSv § 2 Satz 1 KSVG ausübt, die ihre Wurzeln im Ausland oder in einer fremdländischen Kultur hat (vgl. etwa BSG SozR 4-5425 § 2 Nr. 3 - japanische Teezeremonie). Weitere Beispiele verdeutlichen dies: So kann etwa einer musikalischen Darbietung die Anerkennung als "Musik-Kunst" nicht deshalb versagt werden, weil es sich um fremdländische Instrumente, Texte oder Melodien handelt; entsprechend werden auf Kunstausstellungen oder in Museen seit jeher auch Exponate ausländischer bildender Künstler gezeigt und so bewusst auf die Auseinandersetzung mit fremden Sichtweisen oder Darstellungsformen Wert gelegt, ohne dass wegen der Herkunft der Künstler oder ihrer Kunstwerke ernsthafte Zweifel am Merkmal „Bildende Kunst“ bestünden. Daraus folgt, dass für die Beurteilung einer Betätigung als künstlerisch im Sinne des KSVG ausschließlich danach zu fragen ist, ob es sich um eine nach dem Regelungszweck des KSVG und seiner historischen Entwicklung schutzwürdige berufliche Tätigkeit handelt, sie also von einer in § 2 Satz 1 KSVG näher bezeichneten Person ausgeübt wird, der nach der Intention des Gesetzgebers der besondere soziale Schutz des KSVG zukommen soll. Dabei können zur Abgrenzung der künstlerischen von nichtkünstlerischer Betätigung auch die Ursprünge der fraglichen Tätigkeit im Ausland nachzuvollziehen sein. Gleichgültig ist jedenfalls, ob die zu beurteilende Leistung deutsche oder fremdländische Wurzeln hat, ob sie von einem Deutschen oder einem Ausländer dargeboten wird oder ob die ausländischen Bezüge stärker sind als die inländischen - maßgeblich ist allein, dass es sich bei der zu beurteilenden Leistung um Kunst im Sinne des KSVG handelt.

d) Mit dem aus dem Griechischen (kalligraphia) stammenden, aus den Wörtern Schönheit (kállos) und Schreiben (gráphein) zusammengesetzten Begriff der Kalligrafie ist die „Kunst“ des Schönschreibens von Hand, mit Federkiel, Pinsel, Tinte oder anderen Utensilien bezeichnet. Ihr kann kulturell Bedeutung zunächst dort zugekommen sein oder noch zukommen, wo das Abschreiben heiliger Texte ein sakralerVorgang war oder ist. Daneben kam der Kalligrafie bis zum Auf- kommen der Druckerkunst eine zentrale Rolle bei der Übermittlung von Literatur zu. Schließlich entwickelte sich im arabischen und im ostasiatischen Raum eine weitere Bedeutungsebene insoweit, als sie neben der Überlieferung der Schrift auch zu einem Schmuckelement wurde. Im arabischen Raum kam dem wegen des Bilderverbots im Islam eine besondere Rolle zu, weil die Schrift infolgedessen oftmals das einzige Dekor eines Gegenstands enthielt. So entwickelte sich eine Vielzahl von Schriften, die Grundlage eigenschöpferischer, vom Schriftzeichen gelöster und deshalb über die Funktion des Schreibens als bloße Wiedergabe eines vorgefertigten Textes hinausreichender ästhetischer Darstellungen werden konnten (Der Brockhaus, Kunst, 3. Aufl. 2006 - Stichwort Kalligraphie; vgl. auch http://de.wikipedia.org, Stichworte Kalligrafie, Arabische Kalligrafie und Islamische Kunst). Insbesondere in China stellten sich in Zusammenhang damit Verbindungen zwischen Malerei und Schrift ein, die Kalligrafien zu einem integralen Bestandteil von Malerei machten (vgl. Der Brockhaus, aaO, Stichwort Kalligraphie, Ostasien). Diesen Stellenwert der Kalligrafie in der Kulturgeschichte bezeugen nicht zuletzt zahlreiche Sammlungen und Ausstellungen von kalligrafischen Blättern auch im Inland (vgl. etwa den Ausstellungsband "Geschriebene Welten - Arabische Kalligraphie und Literatur im Wandel der Zeit", hrsg. von Deniz Erduman - 2004/05 - Museum für angewandte Kunst, Frankfurt).

e) Danach entspricht eine Tätigkeit als Kalligraf dem Katalogberuf des Malers oder künstlerischen Grafikers, wenn in der Überlieferung vorgefundene Schriftzeichen in Gemälde aufgenommen werden, wie dies insbesondere im ostasiatischen Raum Vorbilder findet. Ebenso liegt es, wenn vorgegebene Schriftzeichen zum Ausgangspunkt grafischer Darstellungen von eigener Gestaltqualität werden, die das Ergebnis als eigenschöpferische Leistung künstlerischen Schaffens erscheinen lassen. Dies ist z.B. der Fall, wenn Kalligrafie zum Gegenstand von Programmen an Kunsthochschulen wird (vgl. etwa die jährlichen Sommerkurse für Kunst und Design an der HAW Hamburg, Pentiment mit jährlichen Kursen in Kalligrafie, http://www.pentiment.de/frameset/frameset.html). Kalligrafie kann aber auch als (kunst)handwerkliche Tätigkeit zu qualifizieren sein, wenn die manuell-technischen Fertigkeiten zur Schreibung nach vorgegebenen Schriftzeichen im Vordergrund stehen, vergleichbar den in Anlage B zur Handwerksordnung ( $\mathrm{HwO}$ ) aufgeführten Handwerken der Glas- und Porzellanmaler (Abschnitt $1 \mathrm{Nr}$. 36), Vergolder (Abschnitt 1 Nr. 52), Theater- und Ausstattungsmaler (Abschnitt 2 Nr. 9) oder Stoffmaler (Abschnitt 2 Nr. 33). Entsprechendes gilt für die Bearbeitung von Materialien wie Holz, Metall, Stein oder ähnlichen Stoffen (vgl. Urteil des Senats vom 28. Februar 2007 - B 3 KS 2/07 R - Tätowierer, zur Veröffentlichung in BSGE und SozR 4 vorgesehen). Auch für die Meisterprüfung im Graveur-Handwerk wird die Beherrschung u.a. von Kalligrafien vorausgesetzt (vgl § 2 Abs. 2 Nr. 8 Verordnung über das Meisterprüfungsberufsbild und über die Prüfungsanforderungen in den Teilen I und II der Meisterprüfung im Graveur-Handwerk vom 16. November 2005, BGBI. I 2005, 3182). Kalligrafie ist danach der bildenden Kunst i.S. von § 2 Satz 1 KSVG dann zuzuordnen, wenn der Schwerpunkt der Berufsausübung (vgl. BSGE 82, 107, 111 = SozR 3-5425 § 25 Nr. 12 S. 64; BSG SozR 4-5425 $\S 2 \mathrm{Nr} .6 \mathrm{Rn}$. 21) durch kalligrafisches Arbeiten in Gemälden oder Grafiken mit eigener, von der überkommenen Zeichenverwendung abgehobener Gestaltqualität geprägt wird. Dies ist der Fall, wenn 
den hergestellten Kalligrafien überwiegend ein hohes $M a ß$ an eigenschöpferischen Inhalten zukommt, sodass sie als von dem zugrunde liegenden Schriftzeichen losgelöstes eigenes künstlerisches Werk und nicht als bloß handwerkliche Wiedergabe eines in der kulturellen Tradition wurzelnden Schriftzeichens zu bewerten ist; dies ist ggf. durch eine sachverständige Begutachtung zu klären. Liegt der Schwerpunkt der Tätigkeit hingegen nur auf der handwerklichtechnischen Bearbeitung von Materialien wie Holz, Metall, Stein o. ä. oder auf der Herstellung von Gebrauchstexten auf Papier, kommt eine Einordnung der Kalligrafie als Kunst iSd § 2 Satz 1 KSVG nur dann in Betracht, wenn der Urheber trotz seiner Stellung als Kunsthandwerker mit seinen Werken in Kunstkreisen als Künstler anerkannt und behandelt wird (vgl. BSGE 80, 136, $138=$ SozR 3-5425 § 2 Nr. 5; BSGE 82, $164=$ SozR 3-5425 § 2 Nr. 8). In diesem Fall ist vor allem maßgebend, ob der Betroffene an Kunstausstellungen teilnimmt, Mitglied von Künstlervereinen ist, in Künstlerlexika aufgeführt wird, Auszeichnungen als Künstler erhalten hat oder andere Indizien auf seine Anerkennung als Künstler schließen lassen.

Nach den mit der Sprungrevision nicht angreifbaren und deshalb für den Senat bindenden ( $§ 161$ Abs. 4, § 163 SGG) Feststellungen des SG ist der Kläger Künstler im Sinne des KSVG. Die von inm geschaffenen Kalligrafien gehen über die Wiedergabe traditionellen Kulturgutes hinaus. Der Kläger wandelt das jeweilige arabische Schriftzeichen individuell so ab, dass es für den Betrachter nicht mehr eindeutig erkennbar ist. Durch seine kreative Gestaltgebung wird das Schriftzeichen entfremdet und das neu geschaffene Bild oder die neu geschaffene Grafik mit einer eigenständigen künstlerischen Aussage versehen, die über das reine Schönschreiben eines Buchstabens oder Zeichens weit hinaus geht. Der Kläger schafft Werke mit eigener Identität, die sich nicht von denen eines herkömmlichen Malers oder künstlerischen Grafikers unterscheiden.

Er gehört deshalb zu dem durch das KSVG geschützten Personenkreis und ist zumindest seit 2003 Künstler iSd § 2 Satz 1 KSVG. Es kommt deshalb nicht darauf an, ob er an Kunstausstellungen teilnimmt, Mitglied von Künstlervereinen ist oder ob er als Künstler in bestimmten Kunstkreisen Anerkennung gefunden hat. Diese vom SG zusätzlich angestellten Erwägungen wären nur dann erforderlich, wenn der Kläger überwiegend (kunst) handwerklich tätig wäre. Dies ist nach den Feststellungen des SG aber gerade nicht der Fall.

\section{Kunsthändler und Folgerecht}

Bundesgerichtshof, Urteil vom 17. Juli 2008 - I ZR 109/05

1. Kunsthändler iSd § 26 UrhG ist jeder, der aus eigenem wirtschaftlichem Interesse an der Veräußerung von Kunstwerken beteiligt ist. Hierzu zählt auch, wer Sammler und Kunstinteressenten beim Kauf und Verkauf von Kunstwerken berät und hierfür eine von der Höhe des Kaufpreises abhängige Provision beansprucht.

2. Der Auskunftsanspruch des Künstlers gegen den Kunsthändler oder Versteigerer gemäß § 26 Abs. 4 Satz 1 UrhG (F: 10.11.1972) setzt ebenso wie der Folgerechtsanspruch des Künstlers gegen den Veräußerer gemäß $\S 26$ Abs. 1 Satz 1 UrhG (F: 10.11.1972) voraus, dass die Weiterveräußerung zumindest teilweise im Inland erfolgt ist.

3. Unter Weiterveräußerung iSd § 26 UrhG ist nicht allein das dingliche Verfügungsgeschäft, sondern das gesamte, das schuldrechtliche Verpflichtungsgeschäft ebenso wie das dingliche Verfügungsgeschäft umfassende Veräußerungsgeschäft zu verstehen (im Anschluss an BGHZ 126, 252, 259 [BGH 16.06.1994 - I ZR 24/92] - Folgerecht bei Auslandsbezug).

4. Bei Unterzeichnung des Kaufvertrags durch einen Vertragspartner im Inland ist der erforderliche Inlandsbezug gegeben. 5. Die Bestimmung des $§ 167$ ZPO ist grundsätzlich auch in den Fällen anwendbar, in denen durch die Zustellung eine Frist gewahrt werden soll, die auch durch außergerichtliche Geltendmachung gewahrt werden kann (Abgrenzung zu BGH, Urt. v. 11.10.1974 - V ZR 25/73, NJW 1975, 39; Aufgabe von BGH, Urt. v. 10.2.1971 - VIII ZR 208/69, WM 1971, 383, 384 und Urt. v. 21.10.1981 - VIII ZR 212/80, NJW 1982, 172).

\section{Tatbestand}

- Die Klägerin ist die Verwertungsgesellschaft Bild-Kunst. Sie nimmt in Deutschland die urheberrechtlichen Befugnisse der ihr angeschlossenen Urheber an Werken der bildenden Künste wahr; hierzu gehört auch der Folgerechtsanspruch nach § 26 UrhG. Der Beklagte berät gegen Provision Sammler und Kunstinteressenten beim Kauf und Verkauf von Kunstwerken.
Die Klägerin verlangt vom Beklagten Auskunft über die Weiterveräußerung von Originalwerken der bildenden Künste ihr angeschlossener Urheber. Sie begehrt zum einen allgemein Auskunft darüber, welche Werke unter seiner Beteiligung im Jahre 2001 weiterveräußert wurden ( $\$ 26$ Abs. 3 UrhG a.F.). Sie erstrebt zum anderen nähere Auskunft über die Veräußerung der Kunstsammlung Ahlers im Januar 2001 und möchte insoweit den Namen und die Anschrift des Veräußerers sowie die Höhe des Veräußerungserlöses der einzelnen Werke erfahren (§ 26 Abs. 4 UrhG a.F.). 